\title{
Téoros
}

Revue de recherche en tourisme

\section{Vers un tourisme sans barrières}

\section{Initiatives dans la région Asie-Pacifique}

\section{ElizaBeth Foggin}

Volume 22, numéro 3, automne 2003

L’accessibilité, une conquête inachevée

URI : https://id.erudit.org/iderudit/1071342ar

DOI : https://doi.org/10.7202/1071342ar

Aller au sommaire du numéro

Éditeur(s)

Université du Québec à Montréal

ISSN

0712-8657 (imprimé)

1923-2705 (numérique)

Découvrir la revue

Citer cet article

Foggin, E. (2003). Vers un tourisme sans barrières : initiatives dans la région Asie-Pacifique. Téoros, 22(3), 16-19. https://doi.org/10.7202/1071342ar d'utilisation que vous pouvez consulter en ligne.

https://apropos.erudit.org/fr/usagers/politique-dutilisation/ 


\section{Vers un tourisme sans barrières Initiatives dans la région Asie-Pacifique}

\section{ElizaBeth Foggin}

$\Delta$ la suite d'un comportement remarquable de la région Asie-Pacifique, les prévisions du World Travel \& Tourism Council (WTTC, 2002) appuyaient celles de l'Organisation mondiale du Tourisme qui identifiait cette région du monde comme en étant une qui, lors de la prochaine décennie, pourrait s'attendre à une croissance exceptionnelle sur le plan du tourisme international, avec des incidences économiques significatives, en dépit des effets négatifs du 11 septembre 2001 .

Les prévisions à long terme sont maintenant réévaluées à la lumière des conséquences néfastes d'événements subséquents tels que l'épidémie de SRAS en 2003. Mais les résultats du nouveau Baromètre du tourisme mondial de l'OMT (WTO, 2003), instrument de mesure qui fait appel aux services d'un groupe de spécialistes en tourisme du monde entier et qui vise à suivre l'évolution à court terme du tourisme, démontrent une perspective plus optimiste pour le proche avenir et cela, pour toutes les régions, y compris la région AsiePacifique.

La Commission économique et sociale pour l'Asie et le Pacifique (CESAP / ESCAP) des Nations Unies a identifié, au sein de cette croissance prévue d'arrivées et de recettes générées par le tourisme intrarégional et international, un marché particulier pour cette région qui, lui aussi, s'avérera important pour l'avenir, soit celui des personnes à capacité physique restreinte. Ce marché repose sur le déve- loppement d'un « tourisme sans barrières », expression qui signifie la réduction de tout obstacle et de tout danger physique ou non-physique qui pourraient nuire aux expériences et aux activités touristiques des personnes à capacité restreinte.

Avec le vieillissement des populations, le marché des voyageurs " seniors » prendra de plus en plus d'ampleur. À cause de l'incidence croissante de l'incapacité avec l'âge (United Nations, 1993 ; Statistics Canada, 1994 ; Australian Bureau of Statistics, 1998), le tourisme sans barrières pourrait répondre aux besoins de ce marché-là aussi bien qu'à ceux du marché déjà significatif des personnes qui ont une capacité physique restreinte, toutes catégories d'âges confondues. Les produits et services accessibles ou qui présentent un minimum d'obstacles sont recherchés également par des familles qui voyagent avec de jeunes enfants, par exemple. Ainsi, le tourisme sans barrières pourrait s'associer au tourisme familial ou « multigénérationnel ».

Les pays ou les régions qui ont les taux les plus élevés de personnes à capacité physique restreinte sont également ceux où le tourisme sans barrières est déjà reconnu : l'Amérique du Nord, certains pays de l'Europe, l'Australie et la Nouvelle-Zélande. Ces mêmes pays sont les sources les plus importantes du tourisme international vers ou au sein de la région Asie-Pacifique.

Vers la fin de la période 1993-2003, période déclarée par les pays-membres de la CESAP comme étant la Décennie Asie-Pacifique pour les personnes handi- capées, deux initiatives en particulier ont révélé l'importance du tourisme sans barrières pour la région.

Lors d'un atelier de la Conférence AsiePacifique sur le tourisme pour les personnes handicapées (Denpasar, Bali, 24-27 septembre 2000), organisée dans le but de créer un lieu d'échange de connaissances et d'expériences et de définir des politiques et des stratégies multisectorielles destinées à encourager le tourisme sans barrières, les participants ont adopté la Déclaration de Bali sur un tourisme sans barrières pour les personnes handicapées (CBRDTC, 2000).

En soulignant l'importance grandissante des personnes à capacité physique restreinte dans un marché mondial de plus en plus concurrentiel, on a constaté que le tourisme sans barrières pourrait aussi servir d'outil efficace pour rehausser les bénéfices socioéconomiques du secteur touristique, de même que pour développer davantage des pays et des régions de la CESAP.

Entre autres propositions, les signataires de la Déclaration de Bali demandaient aux gouvernements des actions concrètes pour intégrer des personnes à capacité restreinte dans divers secteurs de l'industrie touristique, offrant ainsi un autre véhicule pratique pour allèger la pauvreté.

Le document Promotion of Barrier-Free Tourism for People with Disabilities in the Asian and Pacific Region (Vignuda, 2001) appuie ladite Déclaration en soulignant l'importance du marché. De plus, l'auteur identifie les contraintes majeures 
rencontrées par les personnes à capacité restreinte et décrit les conditions et les priorités nécessaires pour promouvoir un tourisme accessible à tous. À la base de toutes ces propositions, on trouve l'affirmation du droit d'accès aux infrastructures touristiques, aux produits et services, y compris les possibilités et les bénéfices des emplois que pourrait offrir l'industrie touristique : «L'industrie touristique devrait offrir les mêmes choix à tous les consommateurs pour assurer la pleine participation des personnes handicapées et protéger les droits de chacun de voyager avec dignité $»$ (Vignuda, 2001). Cela cadre bien avec l'ensemble des droits de l'Homme dans tous les domaines de la vie, sur les plans économique, civil, politique ou social et culturel.

Étant donné que le tourisme a été reconnu comme une industrie en plein essor dans la région Asie-Pacifique et que les personnes handicapées, les personnes âgées et les familles avec de jeunes enfants constituent des groupes grandissants de consommateurs de produits et de services associés aux voyages, aux sports et aux autres activités de loisir (CBRDTC, 2000), la CESAP a, par la suite, commandité un projet de recherche pour aller encore plus loin dans la promotion du tourisme sans barrières.

Quelques facettes de cette recherche - dont le rapport Cameron, Foggin et Darcy (2003) qui a été présenté à Hanoi en mars 2003 au Mekong Tourism Forum, organisé principalement par la Pacific Asia Travel Association (PATA) - sont discutées brièvement dans cet article.

\section{Objectifs ef méthodologie du projet}

Les quatre objectifs principaux de la recherche sont :

- de démontrer un rational économique du développement du tourisme sans barrières ;

- d'identifier les barrières existantes à la pratique du tourisme ;

- d'identifier des exemples de bonnes pratiques d' « accessibilité », au sein des pays-membres de la CESAP; et
- d'élaborer des lignes directrices à suivre pour mieux promouvoir et développer une industrie touristique sans barrières.

Pour les fins du projet et pour préciser davantage ce que signifie l' « accessibilité » ou le «tourisme sans barrières », ce dernier a été défini comme étant :

Un tourisme qui permet à des personnes à capacité restreinte de fonctionner de manière autonome, avec équité et dignité, grâce à une offre de produits, de services et d'environnements touristiques qui tient compte des dimensions physique, visuelle, auditive et cognitive de l'accès ${ }^{3}$ (définition adaptée de celle du gouvernement de la NouvelleGalles du Sud, 1998 ; Olympic Coordination Authority, 1999).

Les méthodes de recherche utilisées comprenaient : un bref examen de la littérature et de données secondaires ; un questionnaire envoyé aux pays-membres ; une recherche sur le réseau Internet pour trouver des éléments de bonnes pratiques; et une analyse et des recommandations relatives au tourisme sans barrières.

Pour respecter la courte échéance proposée par la CESAP, celle-ci a identifié seulement 26 pays sur les quelque 60 pays-membres ; ces pays ont reçu une lettre expliquant le mandat ainsi qu'un questionnaire traitant de sujets tels que la législation et les définitions officielles ou courantes concernant les personnes avec des incapacités. On demandait aux répondants d'identifier des exemples dans leur pays de «bonnes pratiques » de tourisme sans barrières. Seulement sept membres - Polynésie française, Guam, Hong Kong, Indonésie, Japon, Philippines et Singapour - ont répondu au questionnaire. Par la suite, les chercheurs ont examiné pour chacun des 26 pays identifiés par la CESAP, à l'aide de l'engin de recherche Google, jusqu'à 100 hyperliens associés à des mots tels que disability, disabled, handicap et travel. L'ensemble des exemples de tourisme sans barrières repérés, des questionnaires et des résultats de la recherche
Internet ont été retenus et examinés à la lumière de la définition de l'accessibilité mentionnée ci-dessus.

\section{Le développement touristique dans la région Asie-Pacifique}

Le rapport souligne les statistiques recueillies et analysées par des organisations internationales comme l'OMT et le WTTC ainsi que par des regroupements régionaux comme l'APEC (l'Organisation de coopération économique AsiePacifique) et l'ASEAN (l'Association des Nations d'Asie du Sud-Est). Ces statistiques démontrent l'importance du tourisme en termes du nombre et de la provenance des visiteurs ainsi que du montant de dépenses / recettes associées au tourisme. Les prévisions pour l'an 2012 de la contribution du secteur touristique au produit domestique brut pour les diverses régions y sont étalées.

Même si la région Asie-Pacifique n'enregistre pas les chiffres d'arrivées et de recettes les plus élevés des régions du monde, tout indique, pourtant, qu'elle dominera le taux de croissance économique mondiale pour l'industrie touristique.

Une stratégie qui permettrait d'atteindre les prévisions serait de cibler les pays ou les régions qui, traditionnellement, n'envoient que peu de touristes vers l'AsiePacifique. Une autre stratégie serait de cibler un marché-niche avec un réel potentiel comme source nouvelle d'arrivées touristiques. Le tourisme sans barrières représente justement ce genre de créneau.

Les liens entre l'impératif économique et le tourisme sans barrières deviennent évidents. De plus, les bonnes pratiques d'un tel tourisme rejoindront les besoins d'un secteur grandissant de la population tout en respectant les droits humains.

\section{Contraintes et barrières à l'accessibilité}

Après avoir décrit les tendances et les enjeux du tourisme dans la région AsiePacifique, le rapport souligne le fait que, même si les personnes à capacité physique restreinte ont la même gamme de 
motivations pour voyager que la population en général, les barrières pour le faire les affectent de façon disproportionnée (Smith, 1987 ; Kennedy et al., 1991 ; Foggin, 2001).

Les barrières examinées plus particulièrement dans le rapport comprennent celles qui traitent des aspects économiques, de la planification du voyage, de l'utilisation des services des agents de voyages, de la recherche d'informations fiables par des moyens électroniques, du transport, de l'hébergement et de l'accès aux attraits et aux événements touristiques.

Il est question aussi des attitudes des fournisseurs de biens et de services ainsi que des stéréotypes du grand public concernant les incapacités, car le tourisme, en tant que phénomène socioculturel et en tant qu'industrie, s'intègre dans un contexte culturel unique à chaque pays ou région. Les croyances ou les attitudes face aux incapacités ou aux « différences » affecteront l'expérience touristique et, par extension, les stratégies propices à développer davantage le tourisme sans barrières.

Les besoins d'accès varient selon le type d'incapacité. Darcy (1998) en mentionne trois, dont l'accès physique pour les personnes qui se servent, par exemple, des aides pour faciliter leur mobilité. L'accès sensoriel peut être nécessaire pour ceux et celles qui ont une déficience visuelle ou auditive, alors que d'autres personnes ont besoin, pour une raison ou une autre, d'aide pour mieux communiquer avec les autres.

\section{Accès par le mode électronique}

Le réseau Internet sert de véhicule important de communications, en permettant l'accès facile et rapide à une multitude d'information. Ainsi, les chercheurs ont examiné les sites informatisés de divers bureaux nationaux de tourisme pour évaluer leur accessibilité aux touristes à capacité restreinte potentiels. Seulement 8 pays sur les 26 à l'étude offraient des protocoles d'accès reconnus.

Dans la même phase du projet de recherche, d'autres renseignements touristiques

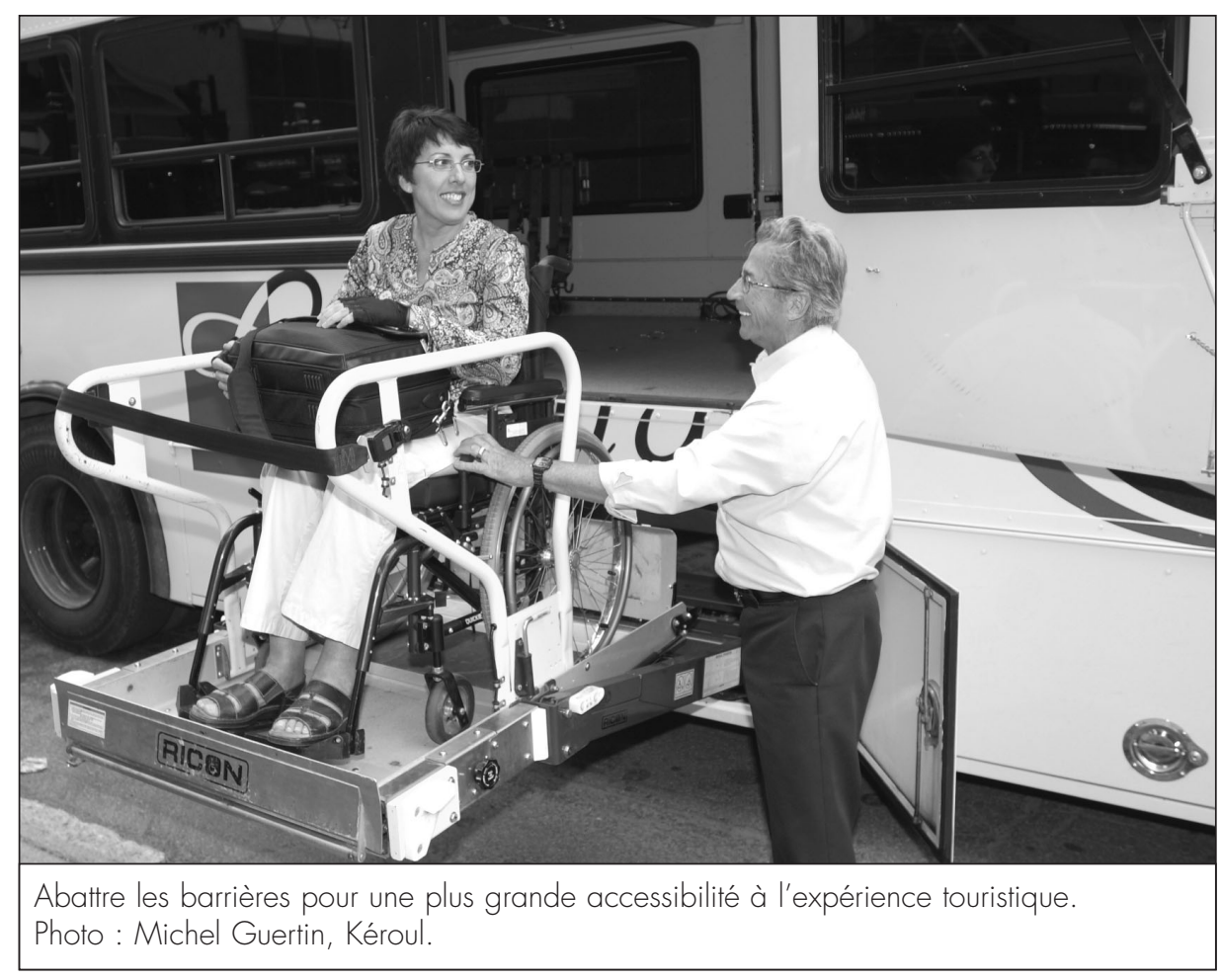

disponibles électroniquement ont été examinés et analysés pour déceler des éléments de bonnes pratiques touchant l'accessibilité. Des exemples dans plusieurs catégories, dont des guides et des cartes d'accès, diverses formes de transport, divers styles d'hébergement, certaines activités et des services de touropérateurs, ont été retenus comme exemples de bonnes pratiques.

\section{Recommandations et conclusions}

Après avoir identifié des exemples et des éléments de bonnes pratiques en tourisme ${ }^{4}$, les auteurs offrent, à $1^{\prime}$ attention des pays, des régions ou des fournisseurs de produits et de services touristiques, des recommandations et des suggestions concrètes pour mieux développer une expérience de tourisme accessible ou sans barrières. Elles concernent des éléments de l'expérience touristique tels que l'infrastructure, le transport, l'hébergement, les activités, l'information, les enjeux économiques ainsi que les conceptions et les barrières culturelles.

Si les personnes à capacité physique restreinte constituent un marché potentiel grandissant de consommateurs de produits et de services de loisirs, dont le tourisme, il est dans l'intérêt des gouvernements et des fournisseurs de ces produits et services de comprendre et d'apprendre comment mieux répondre à leurs besoins.

L'importance de l'accessibilité aux multiples facettes de l'expérience touristique est de mise. De plus, la législation de la part des gouvernements, l'appui actif des diverses organisations pour personnes handicapées ainsi que la formation des intervenants et leur sensibilisation aux besoins des clients potentiels à capacité restreinte demeurent, eux aussi, des enjeux qui requièrent une attention immédiate (Vignuda, 2001).

Cette recherche exploratoire démontre que déjà une offre touristique pour tous se développe tranquillement au sein des marchés touristiques traditionnels de la région AsiePacifique. Soulignons, par exemple, le marché « senior» en Australie (Queensland Office of Ageing, 1998). Examiné de plus près dans le contexte du vieillissement rapide des populations occidentales dû aux «baby boomers», l'argument d'ordre économique est encore plus fort. Il se peut que les événements mondiaux récents aient pu ralentir 
le progrès, mais ils n'ont certes pas anéanti le défi lancé par les initiatives déjà amorcées dans la région Asie-Pacifique pour la promotion d'un tourisme sans barrières.

ÉlizaBeth Foggin a rédigé une thèse de doctorat sur l'expérience touristique des personnes à capacité physique restreinte (Université de Montréal, 2001). Consultante dans l'industrie touristique depuis de nombreuses années, elle est également chargée de cours au Département de géographie de l'Université de Montréal. Le rapport de recherche a été rédigé conjointement par Bruce Cameron (auteur principal du rapport et consultant pour l'industrie touristique en matière d'accessibilité), Simon Darcy (enseignant en tourisme à l'University of Technology et consultant) et ElizaBeth Foggin.

\section{Notes}

1 On peut prendre connaissance de la Déclaration de Bali à l'adresse [http:// unescap.org/decade/baliconferencfinrpt 26.htm].

2 Traduction libre.

3 Traduction libre.

4 Parmi ces exemples, des guides d'accès traitent de multiples facettes de l'expérience touristique (en Australie [www. easyaccessaustralia.com.au] ; en Nouvelle-Zélande [www.travelaxess. co.nz] ; au Japon [www.wakakoma.org] ; à Hong Kong [http://www.hkcss.org.hk/rh/acces sguide/default.htm]) ; des mesures qui favorisent l'accessibilité au transport (en Corée, sur l'accessibilité du métro, voir [http://www.seoulsubway.co.kr/english_s ubway/e_subway05.htm] ; en NouvelleZélande, sur les taxis accessibles, [www.taxinet.org/Links/WebLinks.asp]); de nombreux exemples d'hébergement de toutes catégories (en Australie, l'auberge de jeunesse de Sydney [www. yha.org.au] ; le Tropicana à Cairns où chacune des sept chambres sont adaptées avec sanitaires privés [www.auscyber. net/yal/tropicana.html] ; en Thaïlande, un gîte particulièrement intéressant pour ses services [http://members.ams.chello.nl /danbloker/e_home.html]), des attraits ou des activités touristiques ainsi que des services spécialisés de voyagistes (en Australie, le SeeMore Scenic Tours offre des tours guidés dans un véhicule accessible [www.geotics.com/seemoretours/]; en Asie : Accessible Journeys inc. [www. disabilitytravel.com/aj_details/travel_ agent.htm]).

Le rapport complet (167 pages en anglais) avec de nombreux exemples de bonnes pratiques et de recommandations devrait être bientôt disponible sur le site [http://unescap.org] ; mais on peut déjà l'obtenir en faisant la demande auprès d'ElizaBeth Foggin à beth.foggin@sympa tico.ca.

\section{Bibliographie}

Australian Bureau of Statistics (1998), Disability Ageing and Carers Summary Of Findings (Cat no 4430.0), Australian Bureau of Statistics, Canberra.

Cameron, Bruce, ElizaBeth Foggin, et Simon Darcy (2003), Barrier-Free Travel for People with Disabilities in the Asia Pacific, Recherche / rapport commandité par CESAP, Easy Access Australia Pty Ltd., Kew, Victoria.

Community Based Rehabilitation Development and Training Centre (CBRDTC) (2000), The Bali Declaration on Barrier-free Tourism for People with Disabilities, CBRDTC, Bali, Indonesia [http://unescap.org/ decade/baliconferencfinrpt26.htm].

Darcy, Simon (1998), Anxiety to Access: The Tourism Patterns and Experiences of New South Wales People With a Physical Disability, Tourism New South Wales, Sydney.

ESCAP / CESAP (2002), Asian and Pacific Decade of Disabled Persons 1993 - 2002, United Nations - Economic and Social Commission for Asia and the Pacific [http://www.unescap.org/decade/ (consulté le 20 décembre 2002).

Foggin, ElizaBeth (2001), The Experience of Leisure Tourism of People with Disabilities, Thèse pour l'obtention d'un Ph.D. en Sciences humaines appliquées, Université de Montréal. Kennedy, Dan W., Ralph Smith, et David R Austin. (1991), Special Recreation: Opportunities for Persons With Disabilities (2nd ed.), Wm. C. Brown Pub., Dubuque, Iowa.

New South Wales Government (1998), Disability Policy Framework. Ageing and Disability, Department NSW Health New South Wales Government, Sydney.

Olympic Co-ordination Authority (1999), Access Guidelines ( ${ }^{\text {rd }}$ ed.), Olympic Co-ordination Authority, Sydney.
Queensland Office of Ageing (1998), Not Over the Hill - Just Enjoying the View: A Close-Up Look at the Seniors Market for Tourism in Australia, Seniors Card Tourism Scheme Office of Ageing, Department of Families, Youth and Community Care, Brisbane.

Smith, Ralph (1987), « Leisure of Disabled Tourists: Barriers to Participation », Annals of Tourism Research, vol. 14, nº 3, p. 376-389.

Statistics Canada (1994), Selected Characteristics of Persons With Disabilities Residing in Homes, Health and Limitations Survey.

United Nations (1993), Demographic Yearbook Special Issue: Population Ageing and the Situation of Elderly Persons, United Nations, New York.

Vignuda, Jean-Louis (2001), Promotion of Barrier-Free Tourism for People with Disabilities in the Asian and Pacific Region, Economic and Social Commission for Asia and the Pacific (ESCAP), Bangkok.

World Tourism Organization (2002), Tourism Proves as a Resilient and Stable Economic Sector (Communiqué), World Tourism Organization [http://www.worldtourism.org/ newsroom/Releases/more_releases//june2002/data. htm] (consulté le 10 décembre 2002).

World Tourism Organization (2003), Le premier baromètre du tourisme mondial de l'OMT indique une amélioration régulière de la situation du tourisme international, (Communiqué, Madrid, 30 juin 2003.) [World Tourism Organization [http://www.world-tourism.org/ francais/newsroom/Releases/2003/juin/baro metre.htm] (consulté le 16 septembre 2003).

World Travel and Tourism Council (2002a), Asia-Pacific Destinations Dominate Forecasts for Travel \& Tourism (April 16, 2002 communiqué, World Tourism Organization [http://www.wttc.org/resource Centre/media Centre/releases/020416\%20Asia-Pacific $\% 20$ Destinations\%20Dominant.asp] (consulté le 20 décembre 2002).

World Travel and Tourism Council (2002b), Tourism Satellite Accounts - Impact of Travel and Tourism on Jobs and the Economy Plus Special Report on September $11^{\text {th }}$ Impacts, World Travel and Tourism Council [http:// www.wttc.org/resourceCentre/]. 